\title{
EFFECT OF IRRIGATION TREATMENTS AND SOME SOIL AMENDMENTS ON SOIL PROPERTIES AND PRODUCTION OF WHEAT- PEANUT ROTATION IN SANDY SOIL
}

\author{
Enshrah I.M. El-Maaz ； Hoda M.R.M. Ahmed ;
}

E. M. Aly and Wafaa M.T. El-Etr

Soils, Water and Environ. Res. Instit. Agric. Res. Center (ARC).

Key Words: compost, farmyard manure, polyacrylamide, water use efficiency, wheat, peanut, soil properties.

\section{ABSTRACT}

The use of organic and synthetic soil amendments such as compost, farmyard manure and polyacrylamide can be considered as a specific management to improve the soil physical and chemicals properties of sandy soil along with decreased irrigation water consumptive use and water use efficiency. So, a field experiment was carried out at the Farm of El-Ismailia Agricultural Research Station, El-Ismailia Governorate Egypt in winter season 2014/2015 cultivated with wheat (Triticum aestivum L., cv Giza 168 ) under three water deficit at (100\%, 75\% and $50 \%$ of crop evapotranspiration, ETc) and soil amendments (none, compost, farm yard manure and polyacrylamide). Also, the effect of allowable soil moisture depletion (ASMD) at 25\%,50\% and $75 \%$ of total soil available water was studied on peanut crop (Arachis hypogaea $\mathrm{L}$. Giza 6) under the same previous soil amendments. Wheat and peanut water consumptive use, water use efficiency and both yields components along with physical and chemicals properties of studied sandy soil were also evaluated.

Results indicated that, the highest actual irrigation treatment was recorded at rate of $100 \%$ (ETc) treatment, for wheat crop, while the highest one was recorded under 25\% (ASMD) for peanut crop as compared to other irrigation treatment. Also, the obtained results show a noticeable reduction in soil $\mathrm{pH}$ and salinity as a result of treating the soil with different soil amendments compared to control. The effect was more obvious in case of applying FYM and irrigation treatments $100 \%$ ETc for wheat and 25\% ASMD for peanut crops as compared to other treatments and control. Also, OM and CEC values were increased in case of used FYM soil amendment as compared with other treatments and control for both studied crops under different irrigation treatments. However, the highest diameters of dry aggregates were positive affected by FYM and irrigation treatments $100 \%$ ETc for wheat and 25\% ASMD for peanut crops as compared with other treatments and/or control. In addition, the values of soil bulk density of soil profiles treated by all treatments were relatively low compared to those of control, whereas the maximum 
decrease exists in soil treated by FYM and irrigation treatments $100 \%$ ETc for wheat and 25\% ASMD for peanut crops as compared with other treatments and control. The same trend was true in case of the soil total porosity values. It is clear that application of all treatments decreased soil hydraulic conductivity $\left(\mathrm{cm} \mathrm{h}^{-1}\right)$ values when compared to the control. Moreover, the best treatment in decreasing soil hydraulic conductivity $\left(\mathrm{cm} \mathrm{h}^{-1}\right)$ value of FYM and irrigation treatments $100 \%$ ETc for wheat and 25\% ASMD for peanut crops as compared with other treatments and control. Whereas the highest values of field capacity and available water existed in case of the same treatments.

Finally, applying FYM and irrigation treatments 100\% ETc for wheat and $25 \%$ ASMD for peanut crops as compared with other treatments and control increased significantly the yield and yield components of both wheat and peanut. The beneficial effects of the applied treatments on wheat and peanut yields could be arranged in the following order: FYM>compost > polyacrylamide>control under different irrigation treatments.

\section{INTRODUCTION}

It is of utmost importance to identify the crop production that can be achieved from the basic water unit relative to the cultivated area unit currently and in the future. This is needed because the world population increases especially in developing countries which consequently necessitate increasing food production. However, these increasing trends are not accompanied by similar increase in the fresh available water for everybody. So, the aim of this study is to show the effect of irrigation water deficit and irrigation water stress on wheat and peanut crops production in rotation, respectively.

Rizk and Sherif (2014), Taha et al. (2017) and Morsy et al. (2018) indicated that exposing durum wheat to deficit levels from $60 \%$ to $100 \%$ caused a decrease in all measured parameters in Toshka conditions, Egypt. Ouda et al. (2010) stated that the deficit irrigation till $70 \%$ of full irrigation produced $5 \%$ losses of wheat yield. Zaman et al. (2017) found that water deficit from $60 \%$ to $80 \%$ of field capacity decreased grain yield and water use efficiency of wheat by $15.66 \%$ and 38\%, respectively. Jongrungklang et al. (2008) added that decreased the irrigation amount at levels of field capacity $(100 \%, 25 \%, 40 \%$ and $60 \%$ ) caused drop of peanut water use efficiency. El-Boraie et al. (2009) showed that irrigation quantity $(983.73 \mathrm{~mm})$ produced the highest peanut pods in shalatien sandy soil along with Abd El-Halim et al. (2016) observed that peanut production was $1.32 \mathrm{~kg} / \mathrm{m}^{3}$ at irrigation depth 730 $\mathrm{mm}$ under sprinkler irrigation. Tojo Soler et al. (2013) and Aly et al. (2016) reported that medium water stress level gave the highest peanut water use efficiency. 
Moreover, sandy soil has poor physical and chemical properties including water holding capacity, loose structure, high bulk density and water conductivity, low cation exchange capacity and organic matter. These soils were the main reclaim land in Egypt. Hence, this investigation was carried out on sandy soil, which needs to improve its properties by adding amendments, which was the second goal of this study. Gopinath et al. (2008) found that the organic amendments in sequence, farm yard manure was better than vermicomposting in terms to wheat growth and yield and improved soil properties. Ghosh et al. (2006) and Zayton et al. (2014) showed that straw mulching decreased peanut water consumptive use. Bulluck et al. (2001), El-Hady et al. (2012) and Allam (2017) observed that organic compost and hydrogel conditioners have a good effect on the sandy soil moisture characteristic and crops yield. Singh et al. (2019) stated that water deficit and soil amendment were considered saving water techniques to overcome the water shortage that can be used in agriculture and this was similarly noted by Shenglan et al. (2020).

From the earlier detailed information, the aim of this experiment is to evaluated the consequence of irrigation treatments $(100 \%, 75 \%$ and $50 \%)$ of wheat crop evapotranspiration $\left(\mathrm{ET}_{\mathrm{c})}\right.$ and $25 \%, 50 \%$ and $75 \%$ from available soil moisture depletion (ASMD) irrigation regime for peanut crop in sandy soil treated with some organic and synthetic soil amendments. Water consumptive use, water use efficiency, yields production along with soil physical and chemical properties was taken in consideration.

\section{MATERIALS AND METHODS}

The existing investigation was carried out at the farm of Ismailia Agricultural Research Station in Ismailia Governorate, Egypt, during the winter season (2014/2015) cultivated with wheat (Triticum aestivum L., cv Giza 168) and peanut crop (Arachis hypogaea L. Giza 6) in summer season (2015). The research farm is located at $3035,41.9 " \mathrm{~N}$ latitude and 3216 45.8" E longitude. Some soil physical and chemical properties have been performed according to Klute (1986) and Pansu and Gautheyrou (2006). These results were presented in Table (1 -2).

The main objective of this study was to determine the effect of irrigation deficit levels on wheat crop and available soil moisture depletion to peanut crop with applied soil amendments (none, compost, farm yard manure and poly acrylamide) on water consumptive use, yield of both crops, water use efficiency and some other soil properties. 
Table( 1). Physical analysis and moisture constants of the investigated soil.

\begin{tabular}{|c|c|c|c|c|c|c|c|c|c|c|c|}
\hline \multirow{2}{*}{$\begin{array}{l}\text { Soil } \\
\text { depth } \\
\text { cm }\end{array}$} & \multicolumn{4}{|c|}{ Particle size distribution } & \multirow{2}{*}{ Texture } & \multirow{2}{*}{$\begin{array}{c}\text { Bulk } \\
\text { density } \mathbf{g} \\
\mathbf{c m}^{-3}\end{array}$} & \multicolumn{2}{|c|}{$\begin{array}{l}\text { Retained moisture } \\
\text { at field capacity, } v \\
/ \mathrm{v}\end{array}$} & \multicolumn{2}{|c|}{$\begin{array}{c}\text { Retained moisture at } \\
\text { permanent wilting } \\
\text { point }, \mathrm{v} / \mathrm{v}\end{array}$} & \multirow{2}{*}{$\begin{array}{c}\text { Available } \\
\text { moisture } \\
\text { mm/soil } \\
\text { depth }\end{array}$} \\
\hline & $\begin{array}{c}\text { Coarse sand } \\
\%\end{array}$ & $\begin{array}{l}\text { Fine } \\
\text { sand } \\
\%\end{array}$ & $\begin{array}{c}\text { Silt } \\
\%\end{array}$ & $\underset{\%}{\text { Clay }}$ & & & $\%$ & $\begin{array}{c}\mathrm{mm} / 15 \\
\mathrm{~cm}\end{array}$ & $\%$ & $\begin{array}{c}\mathrm{mm} / 15 \\
\mathrm{~cm}\end{array}$ & \\
\hline 0-15 & 67.50 & 26.86 & 3.77 & 1.87 & Sandy & 1.60 & 12.80 & 19.20 & 3.00 & 4.50 & 14.70 \\
\hline $15-30$ & 70.66 & 24.01 & 3.94 & 1.39 & Sandy & 1.62 & 12.20 & 18.30 & 2.80 & 4.20 & 14.10 \\
\hline $30-45$ & 73.55 & 21.12 & 3.87 & 1.46 & Sandy & 1.65 & 7.92 & 11.88 & 2.60 & 3.90 & 7.98 \\
\hline $45-60$ & 85.47 & 10.87 & 2.65 & 1.01 & Sandy & 1.66 & 6.80 & 10.20 & 2.60 & 3.90 & 6.30 \\
\hline Total & & & & & & & & & & & 43.08 \\
\hline
\end{tabular}

Table (2). Chemical analysis of the investigated soil.

\begin{tabular}{|c|c|c|c|}
\hline Parameters & Values & Parameters & Values \\
\hline pH(1.2.5 soil water susp. & 8.12 & OM \% & 0.23 \\
\hline EC dS m ${ }^{-1}$ & 0.50 & $\mathrm{CaCO}_{3} \%$ & $\mathbf{0 . 5 3}$ \\
\hline \multicolumn{2}{|c|}{ Soluble anions in soil paste extract (meq $\left.L^{-1}\right)$} & \multicolumn{2}{|c|}{ Soluble cations in soil paste extract $\left(\mathrm{meq} \mathrm{L}^{-1}\right)$} \\
\hline $\mathrm{CO}_{3}^{-2}$ & - & $\mathrm{Ca}^{+2}$ & 1.20 \\
\hline $\mathrm{HCO}_{3}^{-}$ & 1.50 & $\mathrm{Mg}^{+2}$ & $\mathbf{0 . 5 0}$ \\
\hline $\mathrm{Cl}^{-}$ & 2.01 & $\mathrm{Na}^{+}$ & 2.80 \\
\hline $\mathrm{SO}_{4}^{--}$ & 1.20 & $\mathbf{K}^{+}$ & 0.21 \\
\hline \multicolumn{4}{|l|}{ Macronutrients in soil } \\
\hline Total N \% & 0.06 & Total P \% & 0.04 \\
\hline Available N (meg Kg $\left.{ }^{-1}\right)$ & 21.6 & Available $P$ meg kg- ${ }^{1}$ & 2.85 \\
\hline
\end{tabular}




\section{Climatic condition:}

The meteorological data ,air temperature $\left(\mathrm{C}^{0}\right)$, relative humidity (\%), actual and possible sunshine (hour), solar and extraterrestrial radiation $\left(\mathrm{MJm}^{-2} \mathrm{day}^{-1}\right)$ and wind speed $(\mathrm{m} / \mathrm{sec})$ had been daily recorded (Table 3) at Ismailia Station , Egypt and their general monthly mean values were calculated.

\section{Irrigation system:}

The experiment was irrigated by a solid set triangle sprinkler system. The laterals were spaced $12 \mathrm{~m}$ apart. The sprinklers were spaced 10 meters lateral. Each two laterals and sprinklers have a control valve to adjust the quantity of applied water. The rate of water application was $45.5 \mathrm{~m}^{3} \mathrm{fed}^{-1} / \mathrm{hr}$ (sprinkler discharge $1.3 \mathrm{~m}^{3} / \mathrm{hr}$ at 2.5 bars). The quantity of applied water was exactly controlled with excellent uniform distribution of water. The number of sprinklers per fed. were 35. The application rate $(\mathrm{A})$ is calculated as follows:-

$$
\mathrm{A}=\mathrm{K} \frac{\mathrm{Qs}}{\mathrm{LS}}
$$

Where: $\mathrm{A}=$ Application rate $[\mathrm{mm} / \mathrm{hr}]$, Qs = Discharge of sprinkler [L/min],

$\mathrm{L}=$ The distance between lateral $[\mathrm{m}], \mathrm{S}=$ The distance between sprinklers on lateral $[\mathrm{m}], \mathrm{K}=$ Fraction equal 60

Table (3). The meteorological general monthly mean values data of

\begin{tabular}{|c|c|c|c|c|c|c|c|c|c|c|c|}
\hline \multirow[b]{2}{*}{ Month } & \multicolumn{11}{|c|}{ Parameters } \\
\hline & $\begin{array}{c}\mathbf{T}_{\max .} \\
{ }^{\circ} \mathbf{C}\end{array}$ & $\begin{array}{c}\mathbf{T}_{\min } . \\
{ }^{\mathbf{o}} \mathbf{C}\end{array}$ & $\begin{array}{c}\mathbf{T}_{\text {mean }} \\
{ }^{\circ} \mathbf{C}\end{array}$ & $\underset{\%}{\mathbf{R H}_{\max }}$ & $\begin{array}{c}\mathbf{R H}_{\min .} \\
\%\end{array}$ & $\begin{array}{c}\mathbf{R} \mathbf{H}_{\text {mean }} \\
\%\end{array}$ & $\begin{array}{c}\text { W.S } \\
\mathrm{m} / \mathrm{sec}\end{array}$ & $\begin{array}{c}\mathrm{N} \\
\text { hour }\end{array}$ & $\begin{array}{c}\mathrm{N} \\
\text { hour }\end{array}$ & $\begin{array}{c}\text { Rs } \\
\text { MJm }^{-2} \\
\text { day }^{-1}\end{array}$ & $\begin{array}{c}\text { Ra } \\
\mathbf{M J m}^{-2} \\
\operatorname{day}^{-1}\end{array}$ \\
\hline Jan. & 19.8 & 8.0 & 13.9 & 79.5 & 21.50 & 50.50 & 2.57 & 7.6 & 10.23 & 12.86 & 20.7 \\
\hline Feb. & 20.8 & 8.5 & 14.65 & 78.9 & 18.50 & 48.55 & 2.91 & 8.3 & 10.97 & 16.02 & 25.5 \\
\hline Mar. & 23.7 & 10.5 & 17.1 & 73.0 & 22.50 & 47.75 & 3.24 & 9.1 & 11.8 & 19.83 & 31.2 \\
\hline Apr. & 28.4 & 13.4 & 20.9 & 71.50 & 19.50 & 45.50 & 3.08 & 10.2 & 12.73 & 23.88 & 36.7 \\
\hline May & 32.5 & 17.3 & 24.9 & 70.5 & 21.00 & 45.75 & 3.03 & 11.5 & 13.53 & 26.99 & 40 \\
\hline Jun. & 35.1 & 20.5 & 27.8 & 71.80 & 23.60 & 47.70 & 2.93 & 13.1 & 13.97 & 29.67 & 41.27 \\
\hline Jul. & 36.4 & 22.5 & 29.45 & 75.50 & 26.20 & 50.85 & 2.93 & 12.6 & 13.83 & 28.66 & 40.63 \\
\hline Aug. & 36.5 & 23.2 & 29.85 & 76.80 & 27.50 & 52.15 & 2.47 & 12.2 & 13.13 & 27.13 & 37.97 \\
\hline Sep. & 33.2 & 21.2 & 27.2 & 76.50 & 30.00 & 53.25 & 2.47 & 10.8 & 12.12 & 22.39 & 32.2 \\
\hline Oct. & 30.9 & 18.1 & 24.5 & 77.50 & 21.70 & 49.60 & 2.17 & 10.2 & 11.27 & 19.16 & 27.27 \\
\hline Nov. & 26.3 & 13.6 & 19.95 & 78.90 & 22.50 & 50.70 & 2.37 & 8.8 & 10.43 & 14.66 & 21.83 \\
\hline Dec. & 21.8 & 9.8 & 15.8 & 79.50 & 23.30 & 51.40 & 2.31 & 7.3 & 10.03 & 11.89 & 19.37 \\
\hline
\end{tabular}
Ismailia Station in the year (2014/2015). 


\section{The layout of first experiment:}

The experiment was carried out in split plot design with three replicates. Wheat seeds (Triticum aestivum L., cv Giza 168) were sown in rows $300 \mathrm{~cm}$ long and $15 \mathrm{~cm}$ apart on December 3, (2014). The field was divided into main plot; $72 \mathrm{~m}^{2}$. The dimension of each plot was $3.0 \mathrm{~m}$ in length and $2 \mathrm{~m}$ in width. Each plot includes 13 rows. The main plots consisted of three irrigation treatments, viz. 100, 75, 50\% of wheat crop evapotranspiration, $\mathrm{ET}_{\mathrm{c}}$, respectively. The sub main plots include also three soil amendments (compost at rate of 5 ton fed ${ }^{-1}$, FYM at rate of 10 $\mathrm{m}^{3} \mathrm{fed}^{-1}$ and polyacrylamide $0.2 \%$ ) along with control treatment. All soil amendments were analyzed and results were presented in Table (4 5).These soil amendments were applied on the soil before cultivation. Normal cultural practices were used including: adding $30 \mathrm{~kg} \mathrm{P}_{2} \mathrm{O}_{5} \mathrm{fed}^{-1}$ in form of calcium superphosphate $\left(15 \% \mathrm{P}_{2} \mathrm{O}_{5}\right)$ before sowing and $48 \mathrm{Kg}$ $\mathrm{K}_{2} \mathrm{O}$ fed. ${ }^{-1}$ in form of potassium sulfate. Nitrogen fertilizer was added as ammonium nitrate $(33 \%)$ at rate of $300 \mathrm{~kg} \mathrm{fed}^{-1}$ divided at six equal doses; after sowing in 20 day and after that added every 15 days. The irrigation treatments $(100,75$ and $50 \%$ of ETc) were applied at end of initial stage. The harvest date of wheat was 30/4/2015.

Table (4):- Chemical composition of the soil conditioners used in the experiment

\begin{tabular}{|l|c|c|l|c|c|}
\hline \multicolumn{1}{|c|}{ Parameters } & Compost & FYM & Parameters & Compost & FYM \\
\hline pH(1:10) & $\mathbf{8 . 0 0}$ & $\mathbf{8 . 7 0}$ & C/N ratio & $\mathbf{2 5 . 1 : 1}$ & $\mathbf{1 9 . 8 : 1}$ \\
\hline EC dSm $^{-1}$ & 4.10 & 4.30 & Total- N \% & $\mathbf{0 . 5 9}$ & $\mathbf{0 . 2 4}$ \\
\hline OC \% & 14.8 & 11.7 & Total- P \% & $\mathbf{0 . 4 4}$ & $\mathbf{0 . 2 0}$ \\
\hline OM \% & $\mathbf{2 5 . 5}$ & $\mathbf{2 0 . 1}$ & Total- K \% & $\mathbf{0 . 6 7}$ & $\mathbf{0 . 1 5}$ \\
\hline
\end{tabular}

Table (5):- Some characteristics of anionic polyacrylamide used in the experiment

\begin{tabular}{|c|c|}
\hline Item & Index \\
\hline Molecular formula & $\left(\mathrm{C}_{3} \mathrm{H}_{5} \mathrm{NO}\right) \mathrm{n}$ \\
\hline Appearance & White granular powder \\
\hline Purity & $>92$ \\
\hline Moisture \% & $<9$ \\
\hline pH value( $1 \%$ water solution) & $7.5-9$ \\
\hline Molecular weight(million) & $16-18$ \\
\hline Charge density & High \\
\hline Approx. bulk density & $\mathbf{0 . 8 0}$ \\
\hline Dissolving time(min.) & $<60$ \\
\hline Ionic character & Anionic \\
\hline \multicolumn{2}{|c|}{ Chemical formula for polyacrylamide } \\
\hline 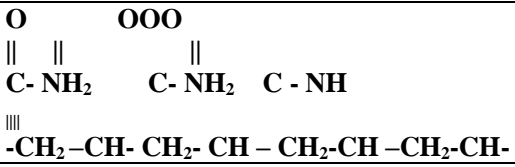 & \\
\hline
\end{tabular}


Wheat evapotranspiration $\left(\mathrm{ET}_{\mathrm{C}}\right)$ calculated by multiplying the Potential evapotranspiration $\left(\mathrm{ET}_{\mathrm{o}}\right)$ and adjusted wheat crop coefficient $(\mathrm{Kc})$ according to the Penman Monteith daily $\left(\mathrm{PM}_{\mathrm{d}}\right)$ equation (Allen et al., 1998).

Where:

$$
\mathrm{ET}_{\mathrm{c}}=\mathrm{K}_{\mathrm{c}} \times \mathrm{ET}_{\mathrm{O}}
$$

$\mathrm{K}_{\mathrm{c}}$ : Crop coefficient.

ETc : The measured (estimate) evapotranspiration of a considered period ( $\mathrm{mm} /$ day)

$\mathrm{ET}_{\mathrm{o}}$ : reference evapotranspiration ( $\mathrm{mm} /$ day) referring to the same period, calculated as average value of formulae.

The duration of wheat crop growth stages were 20, 50, 60 and 23 days for the initial, development, mid-season and late-season, respectively. The adjusted wheat was 0.7 and 0.985 for the initial stage and developmental stages, respectively. While the adjusted wheat crop coefficient $\mathrm{K}_{\mathrm{c}}$ calculated by the next equation were 1.27 and 0.52 for mid-season and late-season a corroding Allen et al. (1998), respectively. $\mathrm{K}_{\mathrm{c} \text { mid }}=\mathrm{K}_{\mathrm{c} \text { mid (Tab) }}+\left[0.04\left(\mathrm{u}_{2}-2\right)-0.004\left(\mathrm{RH}_{\min }-45\right)\right](\mathrm{h} / 3)^{0.3}$ $\mathrm{K}_{\mathrm{c} \text { end }}=\mathrm{K}_{\mathrm{c} \text { end }(\mathrm{Tab})}+\left[0.04\left(\mathrm{u}_{2}-2\right)-0.004\left(\mathrm{RH}_{\min }-45\right)\right](\mathrm{h} / 3)^{0.3}$ Where: $\mathrm{h}=$ plant height, $\mathrm{m}$

The water irrigation management was required at $50 \%$ of the soil waterholding capacity, and also considering the root depth.

\section{The layout of second experiment:}

Peanut (Arachis hypogaea L., cv Giza 6) was planted on $1 / 6 / 2015$. The seeds were placed in holes $25 \mathrm{~cm}$ apart on rows $300 \mathrm{~cm}$ long and $60 \mathrm{~cm}$ between the rows. The experiment was carried out in split plot design with three replicates. The main plot was assigned to irrigation treatments while the sub plot was assigned to soil amendments. The irrigation treatments $(25 \%, 50 \%$ and $75 \%$ of available soil moisture depletion, ASMD) were applied at the end of initial stage. As well as, the same previous mentioned soil amendments with first experiment were applied as sub main plots. Normal cultural practices were used including: adding superphosphate $\left(15 \% \mathrm{P}_{2} \mathrm{O}_{5}\right)$ at rate of $200 \mathrm{~kg} \mathrm{fed}^{-1}$ and potassium sulfate $\left(48 \% \mathrm{~K}_{2} \mathrm{O}\right)$ was applied at rate of $100 \mathrm{Kg}_{\text {fed. }}{ }^{-1}$ divided to equal doses; first one before cultivation and second dose was added to soil after 35 day of sowing date. Nitrogen fertilizer as ammonium nitrate $(33 \%)$ at rate of $100 \mathrm{~kg} \mathrm{fed}^{-1}$. The harvest date of peanut was 8/10/2015.

The irrigation intervals were planned considering the $\mathrm{ET}_{\mathrm{c}}$ and duration for every peanut irrigation treatments. The duration of growth stages for peanut crop are 25, 45, 35 and 25 days for the initial, development, mid-season and late-season, respectively. The adjusted 
peanut coefficients were $0.45,0.75,1.15$ and 0.60 for the initial, developmental mid-season and late-season stages, respectively.

The following characters were included in the study:

1- Water relations:

1.1. Calculation of water consumptive use $(\mathrm{Cu})$ or actual evapotranspiration $\left(\mathbf{E T}_{\mathrm{a}}\right)$ :

Water consumptive use $(\mathrm{Cu})$ was determined according to the equation given by Israelsen and Hansen (1962) as follow:

Where:

$$
W C U=\sum_{i=1}^{n=4} \frac{\left(\theta_{2}-\theta_{1}\right)}{100} \times B d \times D
$$

$\mathrm{WCU}=$ Water consumptive use [mm],

$\mathrm{D}=$ depth of soil layer $(15 \mathrm{~mm}$ each) $[\mathrm{mm}]$,

$\mathrm{Bd}=$ Soil bulk density $\left[\mathrm{g} / \mathrm{cm}^{3}\right]$,

$\mathrm{e}_{1}=$ Soil moisture content before irrigation, $[\mathrm{w} / \mathrm{w}]$,

$\mathrm{e}_{2}=$ soil moisture content after irrigation, $[\mathrm{w} / \mathrm{w}]$.

$\mathrm{n}=$ number of soil layer.

Water use efficiency:

Water use efficiency (WUE) in $\mathrm{kg} / \mathrm{m}^{3}$ was calculated for the deferent treatments, using the following formulae of Zhao et al., 2014):

$\mathrm{Y}$

W.U.E $=--------$

Where: $\mathrm{Y}$ is yields (dry weight, $\mathrm{kg} \mathrm{fed}^{-1}$ ) of a crop

ET is crop water consumption

2- Yield

3- Soil samples:

a. Wheat: straw yield and grain yield $\mathrm{kg}$ fed..$^{-1}$

b. Peanut: straw, pods and seeds yield $\mathrm{kg}$ fed. ${ }^{-1}$

Before planting, soil samples from the surface layer (0-30) have been taken from the experiment site, air-dried, sieved through a $2 \mathrm{~mm}$ sieve and analyzed for some physical and chemical properties. After harvest, undisturbed and disturbed soil samples have been collected from the surface layers (0-30) from all plots for two seasons, air- dried and analyzed for soil $\mathrm{pH}$, organic matter and cation exchange capacity according to the methods described by Page et al. (1982). Particle size distribution was carried out by the pipette method described by Gee and Bauder (1986). The total soluble salts (EC) were determined using electrical conductivity meter at $25^{\circ} \mathrm{C}$ in soil paste extract as $\mathrm{dSm}^{-1}$ (Jackson, 1973). Soil bulk density, total soil porosity and dry aggregates were determined according to Richards (1954). Hydraulic conductivity 
was determined using the undisturbed soil samples according to the method of Richards (1954). Soil moisture equilibrium values were determined according to the methods described by Richards and Weaver (1944) and Richards (1947). Wilting point was determined according to Stakman and Vanderhast (1962), while field capacity was determined as described by Richards (1954).

\section{Statistical analysis:}

All the data collected for the yield and water use efficiency were subjected to the statistical analysis according to Snedecor and Cochran (1980) and the mean values were compared by LSD.

Water relations of two crops:

Wheat actual evaopotranspiration $\left(E T_{a}\right)$ affected by different water treatments and soil amendments.

Results in (Table 6) demonstrate that mean values of wheat $\mathrm{ET}_{\mathrm{a}}$ were $592.75 \mathrm{~mm}, 440.43 \mathrm{~mm}$ and $339.86 \mathrm{~mm}$ at irrigation treatments; $100 \%, 75$ and $50 \%$ of $\mathrm{ET}_{\mathrm{c}}$, respectively. Whereas, the percent 38.45 , 36.48 and $36.88 \%$ of wheat water consumptive use occurred at March for the mentioned irrigation treatments, respectively. This behavior is due to the plant growth stage and weather conditions. Similar results were identified by Rizk and Sherif (2014), Taha et al. (2017) and Morsy et al. (2018). Oweis et al. (2000) added that the seasonal water consumptive use and grain yield varied from $304 \mathrm{~mm}$ to $485 \mathrm{~mm}$ and 170 $\mathrm{g} \mathrm{m}^{-2}$ to500 $\mathrm{g} \mathrm{m}^{-2}$ for wheat in Syria northeast, respectively.

On the other hand, the effect of be relevant different soil amendment; none, compost, farm yard manure (FYM) and polyacrylamide on total mean actual wheat $\left(\mathrm{ET}_{\mathrm{a}}\right)$ results were explained in (Table 6). Mean values of total $\mathrm{ET}_{\mathrm{a}}$ were 489.45, 449.27, 425.71 and $470.04 \mathrm{~mm}$, respectively. The saving water was $8.94 \%, 14.94 \%$ and $4.13 \%$ with utilizing compost, FYM and polyacrylamide, respectively. These results were in agreement with those obtained by Ghosh et al. (2006) and Zayton et al. (2014).

Peanut evaopotranspiration $\left(\mathrm{ET}_{\mathbf{a}}\right)$ affected by different water treatments and soil amendments.

Results in (Table 7) revealed that peanut Eta was $764.13 \mathrm{~mm}$ at $25 \%$ ASMD. Besides, it was $649.195 \mathrm{~mm}$ and $480.61 \mathrm{~mm}$ at 50 and $75 \%$ ASMD, respectively. The highest monthly peanut water consumptive use was achieved at August under different irrigation treatments. The values in percent were 40.99, 35.64 and 29.53 at $25 \%, 50 \%$ and $75 \%$ ASMD, respectively. These results were in agreement with those obtained by ElBoraie et al. (2009) and Abd El-Halim et al. (2016). 
Table (6 ). Wheat daily, monthly and total actual evapotranspiration $\left(\operatorname{ET}_{\mathbf{c}}\right)$ as affected by water deficit and $\quad$ i soil amendments

\begin{tabular}{|c|c|c|c|c|c|c|c|c|c|c|c|c|c|}
\hline \multicolumn{2}{|r|}{ Months } & \multicolumn{2}{|c|}{ Dec." } & \multicolumn{2}{|c|}{ Jan } & \multicolumn{2}{|c|}{ Feb } & \multicolumn{2}{|c|}{ Mar. } & \multicolumn{2}{|c|}{ Apr.** } & \multirow{2}{*}{\multicolumn{2}{|c|}{ Total }} \\
\hline \multirow{2}{*}{$\begin{array}{l}\text { Irrig. } \\
\text { Treat. }\end{array}$} & \multirow{2}{*}{ Soil amendments } & \multirow{2}{*}{$\begin{array}{c}\text { Daily } \\
\text { mm }\end{array}$} & \multirow{2}{*}{\begin{tabular}{|c|} 
monthly \\
mm
\end{tabular}} & \multirow{2}{*}{\begin{tabular}{|c|} 
Daily \\
$\mathbf{m m}$
\end{tabular}} & \multirow{2}{*}{\begin{tabular}{|c|} 
monthly \\
mm
\end{tabular}} & \multirow{2}{*}{$\begin{array}{c}\text { Daily } \\
\text { mm }\end{array}$} & \multirow{2}{*}{$\begin{array}{c}\text { monthly } \\
\text { mm }\end{array}$} & \multirow{2}{*}{$\begin{array}{c}\text { Daily } \\
\text { mm }\end{array}$} & \multirow{2}{*}{\begin{tabular}{|c|} 
monthly \\
mm
\end{tabular}} & \multirow{2}{*}{$\begin{array}{c}\text { Daily } \\
\text { mm }\end{array}$} & \multirow{2}{*}{\begin{tabular}{|c|} 
monthly \\
mm
\end{tabular}} & & \\
\hline & & & & & & & & & & & & $\mathbf{m m}$ & $\mathrm{m}^{3} \mathrm{fed}^{-1}$ \\
\hline \multirow{4}{*}{$100 \% \mathrm{ET}_{\mathrm{c}}$} & None & 2.69 & 75.36 & 3.49 & 108.36 & 4.57 & 128.08 & 7.93 & 245.88 & 2.55 & 76.44 & 634.12 & 2663.3 \\
\hline & Compost & 2.5 & 70.16 & 3.34 & 103.43 & 4.29 & \begin{tabular}{|l|}
120.11 \\
\end{tabular} & 7.12 & 220.62 & 2.29 & 68.68 & 583 & 2448.6 \\
\hline & FYM & 2.48 & 69.38 & 3.00 & 93.03 & 3.91 & 109.41 & 6.86 & 212.79 & 2.17 & 65.05 & 549.66 & 2308.6 \\
\hline & polyacrylamide & 2.58 & 72.12 & 3.37 & 104.38 & 4.35 & 121.81 & 7.5 & 232.5 & 2.45 & 73.4 & 604.21 & 2537.7 \\
\hline \multicolumn{2}{|l|}{ Mean } & 2.56 & 71.755 & 3.3 & 102.3 & 4.28 & 119.85 & 7.35 & 227.94 & 2.36 & 70.89 & 592.75 & 2489.54 \\
\hline \multirow{4}{*}{$75 \% \mathbf{E T}_{\mathrm{c}}$} & None & 2.53 & 70.92 & 2.69 & 83.46 & 3.54 & 99.18 & 5.44 & 168.75 & 1.68 & 50.36 & 472.67 & 1985.2 \\
\hline & Compost & 2.26 & 63.25 & 2.44 & 75.59 & 3.15 & 88.29 & 5.15 & 159.78 & 1.53 & 45.74 & 432.65 & 1817.1 \\
\hline & FYM & 2.11 & 58.94 & 2.27 & 70.36 & 3.04 & 85.14 & 4.7 & 146.77 & 1.44 & 43.2 & 404.41 & 1698.5 \\
\hline & polyacrylamide & 2.33 & 65.15 & 2.61 & 80.88 & 3.22 & 90.11 & 5.4 & 167.46 & 1.61 & 48.39 & 451.99 & 1898.4 \\
\hline \multicolumn{2}{|l|}{ Mean } & 2.3 & 64.565 & 2.5 & 77.57 & 3.24 & 90.68 & 5.18 & 160.69 & 1.56 & 46.92 & 440.43 & 1849.81 \\
\hline \multirow{4}{*}{$50 \% \mathbf{E T}_{\mathbf{c}}$} & None & 2.15 & 60.14 & 2.21 & 68.48 & 2.61 & 73.11 & 4.19 & 130 & 0.99 & 29.83 & 361.56 & 1518.6 \\
\hline & Compost & 1.97 & 55.14 & 1.95 & 60.43 & 2.3 & 64.42 & 4 & 124 & 0.94 & 28.17 & 332.16 & 1395.1 \\
\hline & FYM & 1.97 & 55.14 & 1.94 & 60.02 & 2.13 & 59.58 & 3.89 & 120.72 & 0.92 & 27.61 & 323.07 & 1356.9 \\
\hline & Polyacrylamide & 2.05 & 57.5 & 2.04 & 63.18 & 2.38 & 66.63 & 4.08 & 126.64 & 0.96 & 28.69 & 342.64 & 1439.1 \\
\hline \multicolumn{2}{|l|}{ Mean } & 2.03 & 56.98 & 2.03 & 63.03 & 2.35 & 65.935 & 4.04 & 125.34 & 0.95 & 28.575 & 339.86 & 1427.40 \\
\hline \multirow{4}{*}{$\begin{array}{c}\text { Mean } \\
\text { overall of } \\
\text { soil } \\
\text { amendments }\end{array}$} & None & 2.46 & 68.81 & 2.8 & 86.77 & 3.57 & 100.12 & 5.86 & 181.54 & 1.74 & 52.21 & 489.45 & 2055.7 \\
\hline & Compost & 2.24 & 62.85 & 2.57 & 79.82 & 3.25 & 90.94 & 5.42 & 168.13 & 1.58 & 47.53 & 449.27 & 1886.9 \\
\hline & FYM & 2.18 & 61.15 & 2.4 & $\mathbf{7 4 . 4 7}$ & 3.02 & 84.71 & 5.16 & 160.09 & 1.51 & 45.29 & 425.71 & 1788.0 \\
\hline & Polyacrylamide & 2.32 & 64.92 & 2.79 & 86.58 & 3.316 & 92.85 & 5.66 & 175.53 & 1.67 & 50.16 & 470.04 & 1974.2 \\
\hline
\end{tabular}


Table (7). Peanut daily, monthly and total actual evapotranspiration $\left(\right.$ ET $\left._{\mathrm{a}}\right)$ affected different soil moisture depletion and soil amendments.

\begin{tabular}{|c|c|c|c|c|c|c|c|c|c|c|c|c|c|}
\hline \multicolumn{2}{|l|}{ Months } & \multicolumn{2}{|c|}{ June* } & \multicolumn{2}{|c|}{ July } & \multicolumn{2}{|c|}{ August } & \multicolumn{2}{|c|}{ September } & \multicolumn{2}{|c|}{ October** } & \multirow{2}{*}{\multicolumn{2}{|c|}{ Total }} \\
\hline \multirow{2}{*}{$\begin{array}{l}\text { Irrig. } \\
\text { treat. }\end{array}$} & \multirow{2}{*}{ Soil amendments } & \multirow{2}{*}{$\begin{array}{c}\text { daily } \\
\text { mm }\end{array}$} & \multirow{2}{*}{$\begin{array}{c}\text { monthly } \\
\text { mm }\end{array}$} & \multirow{2}{*}{$\begin{array}{c}\text { daily } \\
\text { mm }\end{array}$} & \multirow{2}{*}{$\begin{array}{c}\text { monthly } \\
\text { mm }\end{array}$} & \multirow{2}{*}{$\begin{array}{c}\text { daily } \\
\text { mm }\end{array}$} & \multirow{2}{*}{$\begin{array}{c}\text { monthly } \\
\text { mm }\end{array}$} & \multirow{2}{*}{$\begin{array}{c}\text { daily } \\
\text { mm }\end{array}$} & \multirow{2}{*}{$\begin{array}{c}\text { monthly } \\
\text { mm }\end{array}$} & \multirow{2}{*}{$\begin{array}{c}\text { daily } \\
\text { mm }\end{array}$} & \multirow{2}{*}{$\begin{array}{c}\text { monthly } \\
\text { mm }\end{array}$} & & \\
\hline & & & & & & & & & & & & $\mathbf{m m}$ & $\mathbf{m}^{3}$ fed $^{-1}$ \\
\hline \multirow{4}{*}{$\begin{array}{c}25 \% \\
\text { ASMD }\end{array}$} & None & 4.16 & 124.7 & 7.12 & 220.8 & 10.92 & 338.71 & 4.11 & 123.41 & 1.96 & 15.66 & 823.28 & 3457.8 \\
\hline & Compost & 4.00 & 120.16 & 6.20 & 192.12 & 9.73 & 301.7 & 3.75 & 112.38 & 1.62 & 13 & $\mathbf{7 3 9 . 3 6}$ & 3105.3 \\
\hline & FYM & 3.90 & 116.99 & 6.03 & 187.11 & 9.71 & 301.03 & 3.60 & 107.96 & 1.5 & 12 & 725.09 & 3045.4 \\
\hline & polyacrylamide & 4.05 & 121.56 & 6.53 & 202.32 & 10.1 & 313.19 & 3.92 & 117.72 & 1.75 & 14 & $\mathbf{7 6 8 . 7 9}$ & 3228.9 \\
\hline \multicolumn{2}{|l|}{ Mean } & 4.02 & 120.85 & 6.47 & 200.59 & 10.12 & 313.66 & 3.84 & 115.37 & 1.71 & 13.66 & 764.13 & 3209.3 \\
\hline \multirow{4}{*}{$\begin{array}{c}\mathbf{5 0 \%} \\
\text { ASMD }\end{array}$} & None & 3.93 & 117.9 & 6.31 & 195.51 & 8.17 & 253.25 & 3.31 & 99.37 & 2.77 & 22.13 & 688.16 & 2890.3 \\
\hline & Compost & 3.89 & 116.72 & 5.86 & 181.8 & 6.93 & 215.02 & 3.13 & 93.98 & 2.13 & 17.02 & 624.54 & 2623.1 \\
\hline & FYM & 3.84 & 115.3 & 5.6 & 176.16 & 6.94 & 215.09 & 3.08 & 92.61 & 2.19 & 17.52 & 616.68 & 2590.1 \\
\hline & polyacrylamide & 30 & 117.02 & 6.15 & 190.7 & 7.81 & 242.25 & 3.29 & 98.61 & 2.35 & 18.82 & 667.4 & 2803.1 \\
\hline \multicolumn{2}{|l|}{ Mean } & 3.89 & 116.73 & 6.00 & 186.04 & 7.46 & 231.40 & 3.20 & 96.14 & 2.36 & 18.87 & 649.195 & 2726.6 \\
\hline \multirow{4}{*}{$\begin{array}{c}\mathbf{7 5 \%} \\
\text { ASMD }\end{array}$} & None & 3.67 & 110.16 & 4.12 & 127.67 & 4.0 & 148.82 & 3.14 & 94.19 & 2.94 & 23.51 & 504.35 & 2118.3 \\
\hline & Compost & 3.62 & 108.49 & 3.83 & 118.65 & 4.51 & 139.88 & 2.95 & 88.63 & 2.54 & 20.39 & 476.04 & 1999.4 \\
\hline & FYM & 327 & 98.27 & 3.70 & 114.83 & 4.33 & 134.11 & 2.88 & 86.55 & 2.5 & 20 & 453.76 & 1905.8 \\
\hline & polyacrylamide & 3.47 & 104.16 & 4.02 & 124.73 & 4.67 & 144.92 & 3.10 & 93.09 & 2.67 & 21.4 & 488.3 & 2050.9 \\
\hline \multicolumn{2}{|l|}{ Mean } & 3.51 & 105.27 & 3.92 & 121.47 & 4.58 & 141.93 & 3.02 & 90.615 & 2.66 & 21.32 & 480.61 & 2018.5 \\
\hline \multirow{4}{*}{$\begin{array}{c}\text { Mean overall } \\
\text { of soil } \\
\text { amendments }\end{array}$} & None & 3.92 & 117.59 & 5.85 & 181.32 & 7.96 & 246.93 & 3.52 & 105.66 & 2.55 & 20.43 & 671.93 & 2822.1 \\
\hline & Compost & 3.84 & 115.12 & 5.30 & 164.19 & 7.06 & 218.87 & 3.28 & 98.33 & 2.10 & 16.80 & 613.31 & 2575.9 \\
\hline & FYM & 3.67 & 110.19 & 5.14 & 159.37 & 6.99 & 216.74 & 3.19 & 95.71 & 2.06 & 16.506 & 598.51 & 2513.7 \\
\hline & polyacrylamide & 3.81 & 114.24 & 5.57 & 172.58 & 7.53 & 233.45 & 3.43 & 103.14 & 2.26 & 18.0 & 641.50 & 2694.3 \\
\hline
\end{tabular}


Moreover, results in (Table 7) show the effect of soil amendments on peanut $\mathrm{ET}_{\mathrm{a}}$. The values of peanut $\mathrm{ET}_{\mathrm{a}}$ ordered from the highest to lowest were as follows: none $(671.93 \mathrm{~mm})$, polyacrylamide $(641.5 \mathrm{~mm})$, compost $(613.31 \mathrm{~mm})$ and farm yard manure $(598.51 \mathrm{~mm})$. Hence, the applied amendments saved water by $4.74 \%$ for poly acrylamide, $9.56 \%$ for compost and $12.27 \%$ for farm yard manure. These results were analogous with Bulluck et al. (2001), El-Hady et al. (2012) and Allam (2017).

Crop yields and water use efficiency affected by irrigation treatments and soil amendments

1- Wheat crop:

Results presented in (Table 8) showed that straw and grains yields and water use efficiency of wheat crop decreased significantly when irrigation depth was decreased from $100 \%$ to $75 \%$ and also from $100 \%$ to $50 \%$ of $\mathrm{ET}_{\mathrm{c}}$, respectively. The reduction in straw, grains and WUE were $26.81 \%, 30.80 \%$ and $7.77 \%$ when irrigation depth dropped from $100 \%$ to $75 \%$. Whereas, the reduction achieved was $58.67 \%, 65.07$ and $39.43 \%$ when irrigation dropped from $100 \%$ to $50 \%$, respectively. These results were in agreement with those reported by Ouda et al. (2010) and Zaman et al. (2017).

Table (8):- Effect irrigation treatments and some soil amendments on wheat crop production in sandy soil

\begin{tabular}{|c|c|c|c|c|}
\hline \multirow[b]{2}{*}{$\begin{array}{l}\text { Irrigation } \\
\text { treatments }\end{array}$} & \multirow[b]{2}{*}{$\begin{array}{c}\text { Type of } \\
\text { amendments }\end{array}$} & \multicolumn{3}{|c|}{ Yield and water use efficiency of wheat } \\
\hline & & $\begin{array}{c}\text { Straw yield } \\
\text { kg fed }^{-1}\end{array}$ & $\begin{array}{c}\text { Grain yield } \\
\text { kg fed }^{-1}\end{array}$ & $\begin{array}{c}\text { WUE } \\
\text { Kg grain } / \mathbf{m}^{3}\end{array}$ \\
\hline \multirow{4}{*}{$\begin{array}{c}100 \% \\
\mathbf{E T}_{\mathrm{c}}\end{array}$} & non & 3100 & 2123 & 0.797 \\
\hline & compost & 3450 & 2387 & 0.975 \\
\hline & FYM & 3567 & 2543 & 1.122 \\
\hline & Poly acrylamide & 3200 & 2373 & 0.935 \\
\hline \multicolumn{2}{|c|}{ Mean for irrigation (I1) } & 3329 & 2356 & 0.9575 \\
\hline \multirow{4}{*}{$\begin{array}{c}75 \% \\
\mathbf{E T}_{\mathrm{c}}\end{array}$} & non & 2283 & 1525 & 0.754 \\
\hline & compost & 2483 & 1683 & 0.926 \\
\hline & FYM & 2603 & 1697 & 0.999 \\
\hline & Poly acrylamide & 2377 & 1618 & 0.852 \\
\hline \multicolumn{2}{|c|}{ Mean for irrigation (I2) } & 2437 & 1631 & 0.883 \\
\hline \multirow{4}{*}{$\begin{array}{c}50 \% \\
\mathbf{E T}_{\mathrm{c}}\end{array}$} & non & 1250 & 703 & 0.463 \\
\hline & compost & 1417 & 897 & 0.641 \\
\hline & FYM & 1487 & 907 & 0.668 \\
\hline & Poly acrylamide & 1350 & 787 & 0.547 \\
\hline \multicolumn{2}{|c|}{ Mean for irrigation (I3) } & 1376 & 823 & 0.580 \\
\hline \multicolumn{5}{|c|}{ Mean for soil conditioners } \\
\hline \multicolumn{2}{|l|}{ Non } & 2211 & 1451 & 0.671 \\
\hline \multicolumn{2}{|l|}{ Compost } & 2450 & 1656 & 0.847 \\
\hline \multicolumn{2}{|c|}{ Farmyard manure } & 2552 & 1716 & 0.930 \\
\hline \multicolumn{2}{|c|}{ Poly acrylamide } & 2309 & 1593 & 0.778 \\
\hline \multicolumn{5}{|c|}{ L.S.D. at $0.5 \%$ for } \\
\hline \multicolumn{2}{|l|}{ irrigation (A) } & 32.28 & 17.03 & 0.011 \\
\hline \multicolumn{2}{|c|}{ Soil amendments (B) } & 35.65 & 14.05 & 0.009 \\
\hline \multicolumn{2}{|l|}{$\mathbf{A} * \mathbf{B}$} & 61.74 & 24.33 & 0.017 \\
\hline
\end{tabular}


Also, results in (Table 8) show that soil amendments; compost, farm yard manure had a significantly increased wheat yield (straw and grains) along with water use efficiency as compared to no applied amendments. These increments in straw yield, grains yield and water use efficiency for wheat crop was $10.8 \%, 14.13$ and $26.21 \%$ when compost was applied. Similarly, increments of $15.43 \%, 18.27 \%$ and $38.49 \%$ and $4.4 \%, 9.81$ and $15.89 \%$ were observed with the addition of farm yard manure and synthesis, respectively. Similar results were found by Gopinath et al. (2008), Leu et al. (2010) and Singh et al. (2019).

\section{2- Peanut crop:}

Peanut straw $\left(\mathrm{kg} \mathrm{fed}^{-1}\right)$, pods $\left(\mathrm{kg} \mathrm{fed}^{-1}\right)$, seeds $\left(\mathrm{kg} \mathrm{fed}^{-1}\right)$ yield and WUE $\left(\mathrm{Kg} \mathrm{seed} / \mathrm{m}^{3}\right)$ were significantly influenced by water stress and the various soil amendments. The obtained values are presented in Table 9. The decreasing ASMD significantly increased peanut straw, pods and seeds production. Whereas, the peanut WUE was produced at medium ASMD. Similar results were found by Tojo Soler et al. (2013), Aly et al. (2016) and Abd El-Halim et al. (2016).

Table (9):- Effect irrigation treatments and some soil amendments on peanut crop production in sandy soil

\begin{tabular}{|c|c|c|c|c|c|}
\hline \multirow[b]{2}{*}{$\begin{array}{l}\text { Irrigation } \\
\text { treatments }\end{array}$} & \multirow[b]{2}{*}{$\begin{array}{c}\text { Type of } \\
\text { amendments }\end{array}$} & \multicolumn{4}{|c|}{ Yield and water use efficiency of peanut } \\
\hline & & $\begin{array}{c}\text { Straw yield } \\
\text { kg fed }^{-1}\end{array}$ & $\begin{array}{l}\text { pods yield } \\
\text { kg fed }^{-1}\end{array}$ & $\begin{array}{l}\text { seed yield } \\
\mathrm{kg} \mathrm{fed}^{-1}\end{array}$ & $\begin{array}{c}\text { WUE } \\
\text { Kg seed } / \mathrm{m}^{3}\end{array}$ \\
\hline \multirow{4}{*}{$25 \%$ ASMD } & Non & 1700 & 1433 & 1103 & 0.323 \\
\hline & Compost & 1927 & 1643 & 1260 & 0.406 \\
\hline & FYM & 2203 & 1787 & 1373 & 0.451 \\
\hline & Poly acrylamide & 1897 & 1648 & 1217 & 0.377 \\
\hline \multicolumn{2}{|c|}{ Mean for irrigation (I1) } & 1932 & 1628 & 1238 & 0.389 \\
\hline \multirow{4}{*}{$\mathbf{5 0 \%}$ ASMD } & Non & 1450 & 1370 & 1003 & 0.347 \\
\hline & Compost & 1597 & 1532 & 1193 & 0.455 \\
\hline & FYM & 1810 & 1632 & 1277 & 0.493 \\
\hline & Poly acrylamide & 1597 & 1533 & 1137 & 0.406 \\
\hline \multicolumn{2}{|c|}{ Mean for irrigation (I2) } & 1613 & 1517 & 1153 & 0.425 \\
\hline \multirow{4}{*}{ 75\% ASMD } & Non & 690 & 542 & 580 & 0.274 \\
\hline & Compost & 850 & 683 & 654 & 0.327 \\
\hline & FYM & 932 & 772 & 693 & 0.371 \\
\hline & Poly acrylamide & 785 & 602 & 567 & 0.276 \\
\hline \multicolumn{2}{|c|}{ Mean for irrigation (I3) } & 814 & 650 & 623 & 312 \\
\hline \multicolumn{6}{|c|}{ Mean for soil conditioners } \\
\hline \multicolumn{2}{|l|}{ none } & 1280 & 1115 & 896 & 0.315 \\
\hline \multicolumn{2}{|l|}{ Compost } & 1458 & 1286 & 1036 & 0.396 \\
\hline \multicolumn{2}{|c|}{ Farmyard manure } & 1648 & 1397 & 1114 & 0.438 \\
\hline \multicolumn{2}{|c|}{ Poly acrylamide } & 1426 & 1261 & 973 & 0.353 \\
\hline \multicolumn{6}{|c|}{ L.S.D. at $0.5 \%$ for } \\
\hline \multicolumn{2}{|l|}{ irrigation $(\mathrm{A})$} & 33.22 & 41.67 & 30.21 & 0.011 \\
\hline \multicolumn{2}{|c|}{ Soil amendments (B) } & 30.56 & 21.41 & 12.92 & 0.009 \\
\hline \multicolumn{2}{|l|}{$\mathbf{A} * \mathbf{B}$} & 52.93 & 37.08 & 22.38 & 0.017 \\
\hline
\end{tabular}


The obtained data for the effect of soil amendments namely; none, compost, farm yard manure and poly acrylamide to peanut straw, pods, seeds and WUE are presented in Table 9. The results revealed that the best soil amendment to peanut production is farm yard manure followed by compost and synthesis, respectively. These results are in agreement with those obtained by Allam (2017) and Shenglan et al. (2020).

Soil properties of the studied soil under wheat- peanut crops.

\section{Soil chemical properties}

Results in Table (10) revealed that soil chemical properties were substantially improved by all treatments. These soil chemical properties included:

\subsection{Soil electrical conductivity:}

Electrical conductivity was a soil parameter that indicates indirectly the total concentration of soluble salts and is a direct measurement of salinity. Soil salinity after harvested wheat and peanut crops as affected by different treatments was given in Table (10). Results showed that slightly increased in EC values as affected by applied irrigation treatments. Applied irrigation treatment 50\% ETc for wheat crop and $75 \%$ ASMD for peanut crop were relatively high EC values as compared to other irrigation treatments for both crops in two successive seasons. In addition, it is clear that application of all treatments significantly decreased soil EC $\left(\mathrm{dSm}^{-1}\right)$ values when compared to control. Shaban et al. (2012) indicated that the decrease of EC soil as treated with applied organic amendments were due to the activity of microorganisms in reducing salinity and simultaneously improving characterization of soil structure; increasing drainable porosity and aggregate stability, and consequently enhanced leaching process through irrigation fractions. The treatment of applied FYM to both studied crops and irrigation treatments $100 \%$ ETc for wheat and 25\% ASMD for peanut has the highest effect in lowering EC values compared with other treatments and control. These results are in agreement with those of Aiad (2010) and Hassan and Abdel Wahab (2013).

\subsection{Soil pH:}

Soil $\mathrm{pH}$ is an important consideration for farmers and graders for several reasons, including the fact that many plants and soil life forms prefer either alkaline or acidic conditions, that some diseases tend to thrive when the soil is alkaline or acidic, and that $\mathrm{pH}$ can affect the availability of nutrients in the soil (Smith et al., 1994). Results of $\mathrm{pH}$ values in Table (10) reveal that no significant different between irrigation 
treatments used in this experiment for both seasons. Also, it is obvious from Table (10) that the soil $\mathrm{pH}$ decreased slightly due to the application of all treatments compared to untreated soil (control) after wheat or peanut harvested. Such decrease in $\mathrm{pH}$ could be attributed to the production of $\mathrm{CO}_{2}$ and organic acids by soil microorganisms acting and other chemical transformation of the added organic matter. The effect was more pronounced in the soil treated with FYM and irrigation treatments (100\% ETc for wheat plant and 25\% ASMD for peanut plant) as compared with other treatments and control. These results are in agreement with Davar et al. (2002) and Rizk (2016) they reported that the soil $\mathrm{pH}$ values decreased in soil treated with FYM. Finally, the reducing of soil $\mathrm{pH}$ as affected by organic amendments application was due to the increase of microbial activity, organic acid production and increase of soil organic matter content compared with control.

\subsection{Soil organic matter and cation exchange capacity:}

Organic matter is regarded as the ultimate source of nutrients and microbial activity in the soil. It is the deciding factor in soil structure, water holding capacity, infiltration rate, aeration and porosity of the soil. Data presented in Table (10) showed that slightly increased in OM content under irrigation treatments (100\% Etc for wheat and 25\% ASMD for peanut) as compared to other irrigation treatments. Moreover, data indicated that the $\mathrm{OM}$ content in soil increased significantly under different treatments and/or control. The highest increase in OM content values was noticed in the treatment of applied FYM and irrigation treatments (100\% ETc for wheat and 25\% ASMD for peanut) as compared with other treatments and control. These results are in agreement with those of El-Eter et al. (2019) who found that the application of compost resulted in increasing of the soil organic matter level.

The cation exchange capacity of the soil as affected by all treatments took the same trend of organic matter. This may be attributed to the soil organic matter which encourages granulation, increases cation exchange capacity (CEC) and is responsible up to $90 \%$ adsorbing power of the soils (Brady and Weil, 2005). Data in Table (10) show that the CEC increased significantly as affected by different treatments compared to control. The highest value of CEC was found in the FYM irrigation treatments (100\% ETc for wheat and 25\% ASMD for peanut) as compared with other treatments and control. Haynes and Naidu (1998) stated that the organic manure caused a $30 \%$ increase in CEC compared with the control treatment. 
Table (10): Chemical properties of the studied soil after wheat- peanut crops harvested

\begin{tabular}{|c|c|c|c|c|c|c|c|c|c|c|c|c|c|c|c|}
\hline \multirow{4}{*}{$\begin{array}{c}\begin{array}{c}\text { Soil } \\
\text { amendments. }\end{array}\end{array}$} & \multicolumn{15}{|c|}{ Wheat crop } \\
\hline & \multicolumn{15}{|c|}{ Irrigation treatments } \\
\hline & \multicolumn{5}{|c|}{$100 \% \mathrm{ET}_{\mathrm{C}}$} & \multicolumn{5}{|c|}{$75 \% \mathbf{E T}_{\mathrm{C}}$} & \multicolumn{5}{|c|}{$\mathbf{5 0} \% \mathbf{E T}_{\mathrm{C}}$} \\
\hline & $\begin{array}{c}\text { EC } \\
\mathbf{d S} \\
\mathbf{m}^{-1}\end{array}$ & $\begin{array}{c}\mathrm{pH} \\
1: 2.5\end{array}$ & $\begin{array}{c}\text { O.M } \\
\%\end{array}$ & $\begin{array}{c}\text { CEC } \\
\text { Cmole/ } \\
\text { kg }\end{array}$ & $\begin{array}{c}\mathrm{CaC} \\
\mathrm{O}_{3} \\
\%\end{array}$ & $\begin{array}{c}\text { EC } \\
\mathbf{d S} \\
\mathbf{m}^{-1}\end{array}$ & $\begin{array}{c}\mathrm{pH} \\
1: 2.5\end{array}$ & $\begin{array}{c}\text { O.M } \\
\%\end{array}$ & $\begin{array}{c}\text { CEC } \\
\text { Cmole/ } \\
\text { kg }\end{array}$ & $\begin{array}{c}\mathrm{CaC} \\
\mathbf{O}_{3} \\
\%\end{array}$ & $\begin{array}{c}\text { EC } \\
\mathbf{d S} \\
\mathbf{m}^{-1}\end{array}$ & $\begin{array}{c}\mathrm{pH} \\
1: 2.5\end{array}$ & $\begin{array}{c}\text { O.M } \\
\%\end{array}$ & $\begin{array}{c}\text { CEC } \\
\text { Cmole/ } \\
\text { kg }\end{array}$ & $\begin{array}{c}\mathrm{CaC} \\
\mathrm{O}_{3} \\
\%\end{array}$ \\
\hline Non & 0.72 & 7.77 & 0.19 & 8.13 & 1.38 & 0.76 & 7.79 & 0.17 & 8.11 & 1.39 & 0.85 & 7.80 & 0.16 & 8.00 & 1.41 \\
\hline Compost & 0.50 & 7.61 & 0.27 & 9.80 & 1.26 & 0.54 & 7.63 & 0.26 & 9.55 & 1.27 & 0.56 & 7.64 & 0.23 & 9.46 & 1.32 \\
\hline FYM & 0.45 & 7.56 & 0.32 & 11.23 & 1.22 & 0.46 & 7.61 & 0.29 & 11.10 & 1.24 & 0.48 & 7.62 & 0.28 & 11.04 & 1.26 \\
\hline PAM & 0.53 & 7.60 & 0.23 & 9.65 & 1.29 & 0.57 & 7.63 & 0.23 & 9.29 & 1.30 & 0.60 & 7.70 & 0.21 & 9.16 & 1.33 \\
\hline
\end{tabular}

\begin{tabular}{|c|c|c|c|c|c|c|c|c|c|c|c|c|c|c|c|}
\hline \multirow{4}{*}{$\begin{array}{c}\begin{array}{c}\text { Soil } \\
\text { amendments }\end{array} \\
\cdot\end{array}$} & \multicolumn{15}{|c|}{ Peanut crop } \\
\hline & \multicolumn{15}{|c|}{ Irrigation treatments } \\
\hline & \multicolumn{5}{|c|}{$25 \%$ ASMD } & \multicolumn{5}{|c|}{ 50\% ASMD } & \multicolumn{5}{|c|}{ 75\% ASMD } \\
\hline & $\begin{array}{c}\text { EC } \\
\mathrm{dS} \mathrm{m}^{-1}\end{array}$ & $\begin{array}{c}\text { pH } \\
1: 2.5\end{array}$ & $\begin{array}{c}\text { O.M } \\
\%\end{array}$ & $\begin{array}{c}\text { CEC } \\
\text { Cmole/ } \\
\text { kg }\end{array}$ & $\begin{array}{c}\mathrm{CaC} \\
\mathrm{O}_{3} \\
\%\end{array}$ & $\begin{array}{c}\mathrm{EC} \\
\underset{1}{\mathrm{dS}} \mathrm{m}^{-}\end{array}$ & $\begin{array}{c}\text { pH } \\
1: 2 . \\
5\end{array}$ & $\begin{array}{c}\text { O.M } \\
\%\end{array}$ & $\begin{array}{c}\text { CEC } \\
\text { Cmole/ } \\
\text { kg }\end{array}$ & $\begin{array}{c}\mathrm{CaC} \\
\mathrm{O}_{3} \\
\%\end{array}$ & $\begin{array}{c}\mathrm{EC} \\
\underset{1}{\mathrm{dS}} \mathrm{m}^{-}\end{array}$ & $\begin{array}{c}\mathrm{pH} \\
1: 2 . \\
5\end{array}$ & $\begin{array}{c}\text { O.M } \\
\%\end{array}$ & $\begin{array}{c}\text { CEC } \\
\text { Cmole/ } \\
\text { kg }\end{array}$ & $\begin{array}{c}\mathrm{CaC} \\
\mathrm{O}_{3} \\
\%\end{array}$ \\
\hline Non & 0.79 & 7.70 & 0.20 & 9.34 & 1.36 & 0.83 & 7.71 & 0.19 & 8.13 & 1.37 & 0.84 & 7.73 & 0.17 & 8.07 & 1.40 \\
\hline Compost & 0.51 & 7.56 & 0.28 & 8.37 & 1.25 & 0.54 & 7.60 & 0.28 & 9.69 & 1.25 & 0.56 & 7.62 & 0.25 & 9.54 & 1.31 \\
\hline FYM & 0.41 & 7.54 & 0.34 & 10.30 & 1.21 & 0.42 & 7.59 & 0.31 & 11.32 & 1.22 & 0.47 & 7.60 & 0.30 & 11.21 & 1.25 \\
\hline PAM & 0.56 & 7.56 & 0.25 & 9.86 & 1.28 & $\mathbf{0 . 5 9}$ & 7.62 & 0.25 & 9.39 & 1.28 & 0.59 & 7.68 & 0.21 & 9.23 & 1.31 \\
\hline
\end{tabular}




\section{Soil physical properties:}

The changes in the studied physical properties of sandy soil as related to the application of all treatments during winter and summer seasons were presented in Table (11 and 12). In general, the studied soil characteristics responded markedly to all the studied treatments, either irrigation or soil amendments, in case of both wheat and peanut crops. Data also indicated that the treatments showed a positive effect for improving the soil characteristics, where, the values of bulk density and hydraulic conductivity decreased, on the other hand, the total porosity and retained moisture at field capacity, wilting point and available water increased as a result of the soil amendment application.

\subsection{Dry -sieved aggregates:}

The dry sieving aggregates values were shown in Table (11). Data reveal that, the dry stable aggregates (D.S.A \%) which having diameters from 1 to $0.5 \mathrm{~mm}$ were found to be the largest size presented in the different studied treatments. Moreover, the percentages of other sizes of dry stable aggregates decrease as their diameters decrease, whereas, the lowest values exist in case of the aggregates having diameters less than $0.063 \mathrm{~mm}$. Thereby, the application of FYM and irrigation treatments $(100 \%$ Etc for wheat and 25\% ASMD for peanut) resulted in the highest increase of diameters 1- 0.5 and 0.5-0.25 mm, compared to control and other treatments. Brian (2015) reported that the relative importance of soil organic matter in maintaining aggregate stability varies with texture. In sandy soils soil organic matter is the most important factor (Oades, 1993).

\subsection{Soil bulk density and total porosity:}

The results obtained in Table (12) showed clearly that the applied organic soil amendments play a dual positive role, i.e., reducing soil bulk density vs increasing total soil porosity. Thus, the promotive effect of organic amendments on the soil porosity in the studied sandy soil may be due to the values of soil bulk density which behaved the opposite trend with those obtained from total porosity. In general, this increase may be related to the increase of storage pores in the studied sandy soil and physical improvement of soil, which can be regarded as an index of an improved soil structure (Amjad et al., 2010). Data also showed that the highest value of total soil porosity was found in the soil treated with FYM and irrigation treatments (100\% ETc for wheat and 25\% ASMD for peanut) compared to control and other treatments. In all treatments, soil bulk density decreased when compared to control, because of binding the primary particles in the aggregates, physically and chemically, and thus in turn increases the stability of the aggregates and limits their breakdown during the wetting process, as a result of applying organic soil conditioners. Generally, organic soil conditioners improve soil physical properties, including improving soil porosity and decreasing soil bulk density. 
Table (11):- Distribution fractions (\%) of dry- sieved aggregates after wheat- peanut crops harvested.

\begin{tabular}{|c|c|c|c|c|c|c|c|c|c|c|c|c|c|c|c|c|c|c|c|c|c|}
\hline \multirow{5}{*}{$\begin{array}{c}\text { Soil } \\
\text { amendment }\end{array}$} & \multicolumn{21}{|c|}{ Wheat crop } \\
\hline & \multicolumn{21}{|c|}{ Irrigation treatments } \\
\hline & \multicolumn{7}{|c|}{$100 \%$ ETc } & \multicolumn{7}{|c|}{$75 \%$ ETc } & \multicolumn{7}{|c|}{$50 \%$ ETc } \\
\hline & \multicolumn{7}{|c|}{ Dry Aggregates Diameter (mm) } & \multicolumn{7}{|c|}{ Dry Aggregates Diameter (mm) } & \multicolumn{7}{|c|}{ Dry Aggregates Diameter (mm) } \\
\hline & 10-2 & $2-1$ & $1-0.5$ & $\begin{array}{l}0.5- \\
0.25\end{array}$ & \begin{tabular}{|c|}
$0.25-$ \\
0.125
\end{tabular} & \begin{tabular}{|l|}
$0.125-$ \\
0.063
\end{tabular} & $<0.063$ & $10-2$ & $2-1$ & $1-0.5$ & $\begin{array}{l}0.5- \\
0.25\end{array}$ & $\mid$\begin{tabular}{l|}
$0.25-$ \\
0.125
\end{tabular} & $\begin{array}{l}0.125- \\
0.063\end{array}$ & $<0.063$ & $10-2$ & $2-1$ & $1-0.5$ & $\begin{array}{l}0.5- \\
0.25\end{array}$ & \begin{tabular}{|l|}
$0.25-$ \\
0.125
\end{tabular} & \begin{tabular}{|c|}
$0.125-$ \\
0.063
\end{tabular} & $<0.063$ \\
\hline Non & 0.93 & 1.56 & 1.75 & 25.60 & 55.64 & 10.78 & 3.58 & 0.82 & 4.25 & 45.0 & 30.80 & 12.88 & 4.97 & 1.28 & 0.92 & 2.31 & 40.76 & 41.88 & 8.30 & 4.29 & 1.54 \\
\hline Compost & 0.73 & 1.63 & 37.79 & 42.07 & 13.94 & 3.33 & 0.51 & 0.46 & 1.32 & 45.20 & 41.64 & 5.82 & 3.65 & 1.91 & 1.00 & 2.60 & 45.08 & 30.17 & 16.94 & 3.40 & 0.80 \\
\hline FYM & 0.48 & 1.36 & 49.44 & 31.84 & \begin{tabular}{|l|}
12.03 \\
\end{tabular} & 4.14 & 0.51 & 0.46 & \begin{tabular}{|l|}
0.94 \\
\end{tabular} & \begin{tabular}{|l|}
43.54 \\
\end{tabular} & 31.67 & \begin{tabular}{|l|}
15.02 \\
\end{tabular} & 6.33 & 2.05 & 1.68 & \begin{tabular}{|l|}
0.98 \\
\end{tabular} & 34.01 & \begin{tabular}{|l|}
45.72 \\
\end{tabular} & \begin{tabular}{|l|}
13.14 \\
\end{tabular} & 4.16 & 0.65 \\
\hline PAM & 1.01 & \begin{tabular}{|l|}
1.25 \\
\end{tabular} & \begin{tabular}{|l}
36.88 \\
\end{tabular} & 38.98 & \begin{tabular}{|l|}
16.11 \\
\end{tabular} & 3.89 & 1.87 & \begin{tabular}{|l|}
0.92 \\
\end{tabular} & 2.56 & 43.20 & 35.25 & \begin{tabular}{|l|}
12.87 \\
\end{tabular} & 3.33 & 2.25 & \begin{tabular}{|l|}
0.86 \\
\end{tabular} & \begin{tabular}{|l|}
2.36 \\
\end{tabular} & \begin{tabular}{|l|}
38.87 \\
\end{tabular} & \begin{tabular}{|l|}
35.99 \\
\end{tabular} & \begin{tabular}{|l|}
14.72 \\
\end{tabular} & 5.47 & 1.74 \\
\hline
\end{tabular}

\begin{tabular}{|c|c|c|c|c|c|c|c|c|c|c|c|c|c|c|c|c|c|c|c|c|c|}
\hline \multirow{5}{*}{$\begin{array}{c}\text { Soil } \\
\text { amendment }\end{array}$} & \multicolumn{21}{|c|}{ Peanut crop } \\
\hline & \multicolumn{21}{|c|}{ Irrigation treatments } \\
\hline & \multicolumn{7}{|c|}{$25 \%$ ASMD } & \multicolumn{7}{|c|}{ 50\% ASMD } & \multicolumn{7}{|c|}{ 75\% ASMD } \\
\hline & \multicolumn{7}{|c|}{ Dry Aggregates Diameter (mm) } & \multicolumn{7}{|c|}{ Dry Aggregates Diameter (mm) } & \multicolumn{7}{|c|}{ Dry Aggregates Diameter (mm) } \\
\hline & 10-2 & $2-1$ & $1-0.5$ & \begin{tabular}{|l|}
$0.5-$ \\
0.25
\end{tabular} & \begin{tabular}{|l|}
$0.25-$ \\
0.125
\end{tabular} & \begin{tabular}{|c|}
$0.125-$ \\
0.063
\end{tabular} & $<0.063$ & $10-2$ & $2-1$ & $1-0.5$ & $\begin{array}{l}0.5- \\
0.25\end{array}$ & \begin{tabular}{|l|}
$0.25-$ \\
0.125
\end{tabular} & $\begin{array}{r}0.125- \\
0.063\end{array}$ & $<0.063$ & $10-2$ & $2-1$ & $1-0.5$ & $\begin{array}{l}0.5- \\
0.25\end{array}$ & \begin{tabular}{|l|}
$0.25-$ \\
0.125
\end{tabular} & $\begin{array}{l}0.125- \\
0.063\end{array}$ & $<0.063$ \\
\hline Non & 0.42 & 1.45 & 26.05 & 56.75 & 9.66 & 3.81 & 1.85 & 0.46 & 4.43 & 41.29 & 33.85 & 12.94 & 5.21 & 1.81 & 0.58 & 1.77 & 40.19 & 45.14 & 8.21 & 5.71 & 2.17 \\
\hline Compost & 0.34 & 1.78 & 33.35 & 44.56 & 13.17 & 4.09 & 2.71 & 0.42 & 1.43 & 43.02 & 43.96 & 5.41 & 4.09 & 1.68 & 0.61 & 2.27 & 41.77 & 34.10 & 16.01 & 3.78 & 1.47 \\
\hline FYM & 0.61 & 1.16 & 37.22 & 43.21 & 11.34 & 4.42 & 2.04 & 0.47 & 1.03 & 38.42 & 38.87 & 13.53 & 5.53 & 2.15 & 0.39 & 0.86 & 32.60 & 47.05 & 12.71 & 4.28 & 2.11 \\
\hline PAM & 0.31 & 1.20 & 36.16 & 43.12 & 12.99 & 4.06 & 2.15 & 0.46 & 2.52 & $\mid 40.09$ & 37.78 & $\mid 12.54$ & 3.85 & 2.76 & 0.65 & 2.52 & 36.42 & 38.81 & 14.08 & 5.40 & 2.12 \\
\hline
\end{tabular}


Table ( 12 ):- Soil moisture constants (\%), total porosity (\%), Hydraulic conductivity and Bulk density after wheat-peanut plants harvested

\begin{tabular}{|c|c|c|c|c|c|c|c|c|c|c|c|c|c|c|c|c|c|c|}
\hline \multirow{5}{*}{$\begin{array}{c}\text { Soil } \\
\text { amendment }\end{array}$} & \multicolumn{18}{|c|}{ Wheat crop } \\
\hline & \multicolumn{18}{|c|}{ Irrigation treatments } \\
\hline & \multicolumn{6}{|c|}{$100 \%$ ETc } & \multicolumn{6}{|c|}{$75 \%$ ETc } & \multicolumn{6}{|c|}{$50 \%$ ETc } \\
\hline & \multirow{2}{*}{$\mid \begin{array}{c}\text { Hydrulic } \\
\text { conductivity } \\
\left(\mathrm{cm} \mathrm{h}^{-1}\right)\end{array}$} & \multirow{2}{*}{$\begin{array}{c}\text { T.P. } \\
\%\end{array}$} & \multirow{2}{*}{$\begin{array}{c}\mathbf{B D} \\
\left(\mathrm{g} / \mathrm{cm}^{3}\right)\end{array}$} & \multicolumn{3}{|c|}{$\begin{array}{l}\text { Soil moisture } \\
\text { constants \% }\end{array}$} & \multirow{2}{*}{$\begin{array}{c}\text { Hydraulic } \\
\text { conductivity } \\
\left(\mathrm{cm} \mathrm{h}^{-1}\right)\end{array}$} & \multirow{2}{*}{$\begin{array}{c}\text { T.P. } \\
\%\end{array}$} & \multirow{2}{*}{$\left|\begin{array}{c}\mathbf{B D} \\
\left(\mathrm{g} / \mathrm{cm}^{3}\right)\end{array}\right|$} & \multicolumn{3}{|c|}{$\begin{array}{l}\text { Soil moisture } \\
\text { constants \% }\end{array}$} & \multirow{2}{*}{$\begin{array}{c}\text { Hydrulic } \\
\text { conductivity } \\
\left(\mathbf{c m ~ h}^{-1}\right)\end{array}$} & \multirow{2}{*}{$\begin{array}{c}\text { T.P. } \\
\%\end{array}$} & \multirow{2}{*}{$\begin{array}{c}\text { BD } \\
\left(\mathrm{g} / \mathrm{cm}^{3}\right)\end{array}$} & \multicolumn{3}{|c|}{$\begin{array}{l}\text { Soil moisture } \\
\text { constants \% }\end{array}$} \\
\hline & & & & F.C. & W.P & A.W. & & & & F.C. & W.P & A.W. & & & & F.C & W.P. & A.W. \\
\hline Non & 11.96 & 34.84 & 1.73 & 12.13 & 7.71 & 4.41 & 12.81 & 32.95 & 1.78 & 12.08 & 8.04 & 4.04 & 13.01 & 31.95 & 1.80 & 12.00 & 8.10 & 3.90 \\
\hline Compost & 9.88 & 40.50 & 1.58 & 15.88 & 5.04 & 10.82 & 9.95 & 40.0 & 1.59 & 15.43 & 5.09 & 10.34 & 9.79 & 38.62 & 1.63 & 15.01 & 5.39 & 9.63 \\
\hline FYM & 8.33 & 48.81 & 1.36 & 18.73 & 4.53 & 14.19 & 8.47 & 48.05 & 1.38 & 18.02 & 4.37 & 13.65 & 8.52 & 46.67 & 1.41 & 17.65 & 4.62 & 13.03 \\
\hline PAM & 9.90 & 37.61 & 1.65 & 13.87 & 5.61 & 8.33 & 10.00 & \begin{tabular}{|l|}
36.48 \\
\end{tabular} & 1.68 & $\mathbf{1 3 . 5 9}$ & 5.77 & 7.82 & 10.01 & 35.98 & 1.70 & 13.59 & 5.02 & 8.57 \\
\hline
\end{tabular}

\begin{tabular}{|c|c|c|c|c|c|c|c|c|c|c|c|c|c|c|c|c|c|c|}
\hline \multirow{5}{*}{$\begin{array}{c}\text { Soil } \\
\text { Amendment. }\end{array}$} & \multicolumn{18}{|c|}{ Peanut crop } \\
\hline & \multicolumn{18}{|c|}{ Irrigation treatments } \\
\hline & \multicolumn{6}{|c|}{ 25\% ASMD } & \multicolumn{6}{|c|}{ 50\% ASMD } & \multicolumn{6}{|c|}{ 75\% ASMD } \\
\hline & \multirow{2}{*}{$\mid \begin{array}{c}\text { Hydrulic } \\
\text { conductivity } \\
\left(\mathrm{cm} \mathrm{h}^{-1}\right)\end{array}$} & \multirow{2}{*}{\begin{tabular}{c|} 
T.P. \\
$\%$
\end{tabular}} & \multirow{2}{*}{$\begin{array}{c}\text { BD } \\
\left(\mathrm{g} / \mathrm{cm}^{3}\right)\end{array}$} & \multicolumn{3}{|c|}{$\begin{array}{l}\text { Soil moisture } \\
\text { constants \% }\end{array}$} & \multirow{2}{*}{$\begin{array}{c}\text { Hydraulic } \\
\text { conductivity } \\
\left(\mathrm{cm} \mathrm{h}^{-1}\right)\end{array}$} & \multirow[t]{2}{*}{$\begin{array}{c}\text { T.P. } \\
\%\end{array}$} & \multirow{2}{*}{$\begin{array}{c}\text { BD } \\
\left(\mathrm{g} / \mathrm{cm}^{3}\right)\end{array}$} & \multicolumn{3}{|c|}{$\begin{array}{l}\text { Soil moisture } \\
\text { constants \% }\end{array}$} & \multirow{2}{*}{$\begin{array}{c}\text { Hydrulic } \\
\text { conductivity } \\
\left(\mathrm{cm} \mathrm{h}^{-1}\right)\end{array}$} & \multirow[t]{2}{*}{$\begin{array}{c}\text { T.P. } \\
\%\end{array}$} & \multirow{2}{*}{$\begin{array}{c}\mathbf{B D} \\
\left(\mathrm{g} / \mathrm{cm}^{3}\right)\end{array}$} & \multicolumn{3}{|c|}{$\begin{array}{l}\text { Soil moisture } \\
\text { constants \% }\end{array}$} \\
\hline & & & & F.C. & W.P. & A.W. & & & & F.C. & W.P. & A.W. & & & & F.C. & W.P. & A.W. \\
\hline Non & 11.92 & 37.48 & 1.66 & 12.25 & 7.12 & 5.13 & 12.76 & 34.09 & 1.75 & 12.12 & 8.01 & 4.11 & 12.30 & 33.20 & 1.77 & 11.65 & 7.93 & 3.72 \\
\hline Compost & 9.81 & 41.90 & 1.54 & 16.73 & 4.91 & 11.82 & 9.89 & 40.38 & 1.58 & 15.46 & 4.92 & 10.54 & 9.94 & 39.50 & 1.60 & 16.27 & 5.3 & 10.97 \\
\hline FYM & 8.22 & 49.68 & 1.33 & 18.81 & 4.45 & 14.36 & 8.28 & 48.68 & 1.36 & 18.33 & 3.8 & 14.53 & 8.34 & 46.92 & 1.41 & 17.92 & 3.73 & 14.19 \\
\hline РАM & 9.79 & 39.25 & 1.61 & 14.09 & 5.43 & 8.66 & 9.86 & 38.24 & 1.64 & 13.54 & 5.45 & 8.09 & 10.01 & 36.73 & 1.68 & 12.81 & 3.55 & 8.07 \\
\hline
\end{tabular}




\subsection{Hydraulic conductivity and soil moisture constants:}

Values of soil hydraulic conductivity after harvested wheat and peanut crops as affected by different treatments are given in Table (12). It is clear that the application of all treatments decreased soil $\mathrm{HC}\left(\mathrm{cm} \mathrm{h}^{-1}\right)$ values when compared to the control. The improvement or the pronounced decrease in hydraulic conductivity of the studied sandy soil may be attributed to the creation of micro pores, and the dominance of meso and micro pores compared with other pore sizes. These results are in agreement with those of El-Fayoumy and Ramadan (2002). The best treatment in decreasing soil $\mathrm{HC}\left(\mathrm{cm} \mathrm{h}^{-1}\right)$ values was FYM compared to control and other treatments.

Concerning the magnitudes of the changes in available water range, field capacity and wilting point at different applied treatments, data presented in Table (12), in general, showed that the content (\%) of available water in soil increased .The soils treated with FYM relatively high values of available water as compared to control and other treatments. This is due to the fact that organic substances attain a pronounced high content of active organic compounds that enhancing the water molecules to be chelated (Moustafa et al., 2005). The highly magnitude of these results is saving a lot of irrigation water which can be used to reclaim, cultivate new areas and to enhance water use efficiency of most crops. These results are in harmony with the findings of Usman et al. (2005) and Hassan and Abdel Wahab (2013).

In general, FYM effect of the applied treatments on the studied different soil physical properties under the application of FYM and irrigation treatments (100\% ETc for wheat and 25\% ASMD for peanut) could be arranged in the following order: FYM> compost> polyacrylamide $>$ control .

\section{CONCLUSION}

From the abovementioned results, it could be concluded that applied irrigation treatments (100\% ETc for wheat and 25\% ASMD for peanut) and used organic and synthetic soil amendments such as compost, farmyard manure and polyacrylamide can improve the soil physical and chemicals properties of sandy soil along with decreased irrigation water consumptive use and increased water use efficiency. Moreover, wheat and peanut yields increased significantly under the irrigation treatment (100\% ETc for wheat and 25\% ASMD for peanut) in presence of FYM soil amendment as compared to other treatments or control treatment. 


\section{ACKNOWLEDGMENT}

The authoresses wish to express their sincere gratitude and appreciation to the Development of Soil Conditioners Project, Dept. of Physics and Chemistry of Soil, Soils, Water and Environ. Res. Inst., Agric. Res. Center (ARC), Giza, Egypt, for introducing all facilities needed to accomplish this study.

\section{REFERENCES}

Abd El-Halim, A. K.; A.M Awad and M. E Moursy (2016) . Response of peanut to some kinds of organic fertilizers under drip and sprinkler irrigation systems. Alex. Sci. Exch. J., 4:703-713.

Allam, A. Kh (2017). Effects of soil conditioner on water content of sandy soil and peanut production under different irrigation rates. Misr J. Ag. Eng., 34 (3): 1271 - 1296.

Allen, R.G. ; L.S. Pereira ; D. Raes and M. Smith (1998). Crop evapotranspiration: guidelines for computing crop water requirements. FAO. Irrigation and Drainage Paper 56. Rome: Food and Agriculture Organization of the United Nations.

Aly, E.M. ; Wafaa M.T. El-Etr and G.H. Youssef (2016). Peanut (Arachis hypogaea L.) response to different levels of irrigation stress and sythsitic soil amendements. Egypt. J. Soil Sci.,56 (2):351- 371.

Amjad, A.S.A; Y.M.A. Khanif; H.A. Aminuddin; O.A. Radziah and H.A. Osumanu (2010). Impact of potassium humate on selected chemical properties of an Acidic soil. 19th World Congress of Soil Science, Soil Solutions for a Changing World 1-6 August 2010, Brisbane, Australia.

Brady, N. C. and R. R. Weil (2005). The Nature and Properties of Soils. 13thEdition. Macmillan Publishing Company, New York. PP. 279-313.

Brian, M. (2015).Key soil functional properties affected by soil organic matter - evidence from published literature .Earth and Environ. Sci., 25:125-138.

Bulluck,L.R. ; M. Brosius ; G.K. Evanylo and J.B. Ristaino (2001). Organic and synthetic fertility amendments influence soil microbial, physical and chemical properties on organic and conventional farms. Appl. Soil Ecol.,19:147-160.

Davar nejad, G.; G. Haghniya; H. Shahbazi and R. Mohammdiyan (2002). Effect of compost and manure in the production of sugar Jghndr. Journal of Agricultural Science and Technology, 16: 75-83. 
El- Boraie, F.M. ; H.K. Abo-El-Ela and A.M. Gaber (2009). Water requirements of peanut grown in sandy soil under drip irrigation and biofertilization. Aust. J. Basic and Appl.Sci., 3: 55-65.

El-Eter, Wafaa M. ; Hoda M.R.M.Ahmed ; Enshrah I.M. EI Maaz and W.M. El-Farghal (2019).The relative effects of different soil conditioners and levels of irrigation on soil properties and plant growth under condions of sandy soil. Egtpt J. of Appl. Sci., 34(1):46-71.

El-Fayoumy, M.E. and H.M. Ramadan (2002). Effect of bio-organic manure on sandy soils amelioration and peanut productivity under sprinkler irrigation system. Egypt. J. Soil Sci., 42(3): 838.

El-Hady, O.A. ; S.M. Shaaban and Sh.A. Wanas (2012). Effect of hydrogels and organic composts on soil hydrophysical properties and on production of tomato. Proc. XXVIIIth IHC IS on Organic Horticulture: Productivity and Sustainability Eds.: I. Mourão and U. Aksoy Acta Hort., 933, ISHS.

Gee, G.W. and J. W. Bauder (1986). Particle size analysis in Methods of Soil Analysis (Klute, Ed. Part1. Agron.9. 15:383- 409. Am. Soc. Agron. Madison. Wisconsin, U.S.A).

Ghosh, P.K. ; K.K. Devi Dayal ; M. Bandyopadhyyay and A. Mohanty (2006). Evaluation of straw and polythene mulch for enhancing productivity of irrigated summer groundnut. Field Crop Res., 99:76-86.

Gopinath, K. A. ;S. Saha ; B. L. Mina ; H. Pande ; S. Kundu and H. S. Gupta (2008). Influence of organic amendments on growth, yield and quality of wheat and on soil properties during transition to organic production. Nutr. Cycl. Agroecosyst., 82:51-60.

Hassan, A. Z. A. and M.M. Abdel Wahab (2013). The combined effect of bentonite and natural zeolite on sandy soil properties and productivity of some crops. Topclass J. of Agric. Res., 1: 22-28.

Haynes, R. J. and R. Naidu (1998). Influence of lime, fertilizer and manure applications on soil organic matter content and soil physical conditions. Nutrient Cycling in Agroecosystems, 51: 123-137.

Israelsen, O.W. and V.E. Hansen (1962). Irrigation Principles and Practices. The 3rd ed. John, Wiley and Sons Inc., New York.

Jackson, M. L. (1973). Soil Chemical Analysis. Prentic Hall of Indian Private Limited, New Delhi, India. 
Kesmala and A. Patanothi (2008).Identification of peaunt genotypeswith high use efficiency under drought stress conditions from peaunt germplasm of diverse origins. Asian J. Plant Sci., 7:628-638.

Klute, A. (1986). Water retention: laboratory methods. In: Klute A, editor. Methods of soil analysis: Physical and mineralogical methods. 2nd ed. Madison: Amer. Soc. of Agron., Soil Soc. Amer.; Pt. 1. p. 635-62.

Leu, J. ; S. Traore ; Y. Wang and C.E. Kan(2010). The effect of organic matter amendment on soil water holding capacity change for irrigation water saving: Case study in Sahelian environment of Africa. Sci.Res. Essays, 5: 3564-3571.

Morsy, A.S.M. ; A. Awadalla and M.M. Sherif (2018). Effect of irrigation, foliar spray with nano-fertilizer (lithovit) and nlevelson productivity and quality of durum wheat under toshka conditions. Assiut J. Agric. Sci., $3:$ 1-26.

Moustafa, M.A. Abo-Zied; N.R. Habashy and A.A.W. Anas (2005). Utilization of some organic polymers and humic acids for improving a sandy soil productivity of peanut and their residual effects on the nex crop of faba bean. Fayoum J. Agric. Res. And Dev., 9(2): 42-55.

Oades, J.M. (1993). The role of soil biology in the formation, stabilization and degradation of soil structure. Geoderma. 56: 377-400.

Ouda, S.A. ; R. Abou Elenin and M. A. Shreif (2010). Simulation of the effect of irrigation water saving on wheat yield at middle egypt. Fourteenth International Water Technology Conference, IWTC 14 2010, Cairo, Egypt, 407-419.

Oweis, T. ; H. Zhang and M. Pala (2000). Water use efficiency of rainfed and irrigated bread wheat in a mediterranean environment. Agron. J., 92:231-238.

Pansu, M. and J. Gautheyrou(2006). Handbook of Soil Analysis. Mineralogical, Organic And Inorganic Methods.

Page, A.L; R. H. Miller and D. R. Keeny (1982). Methods of soil analysis. Part 2- chemical and microbiological properties second Edition Ajner. Soc. of Agron. Madison, Wisconsin, USA. 5371.

Richards, A. L. and I. R. Weaver (1944). Moisture retention by some irrigated soils as related to soil moisture tension. J. Agric. Res., 29:215-235. 
Richards, A. L. (1947). Pressure membrane apparatus construction and Use. Agric. Enger., 28: 451-454.

Richards, A.L. (1954). Diagnosis and Improvement of Saline and Alkali Soils U.S. Dept. Agric. Hand Book. No 60, U.S.Covt. Print. Office, Washington, D.C.

Rizk, A.H. and M.M. Sherif (2014). Effect of soil moisture depletion on the yield of wheat under sprinkler irrigation at Toshka area, Egypt. Middle East J. Agric.Res., 3: 981-987.

Shaban, Kh. A.; M. G. Abd-El-kader and Z. M. Khalil (2012). Effect of soil amendments on soil fertility and sesame crop productivity under newly reclaimed soil conditions. J. of Appl. Sci. Res., 8 (3): 1568 - 1575.

Shenglan,Y. ; T. Liu andY. Niu (2020). Effects of organic fertilizer on water use, photosynthetic characteristics, and fruit quality of pear jujube in northern Shaanxi. Open Chem., 18: 537-545.

Singh, M. ; R. K. Saini and S. Singh (2019).Potential of integrating biochar and deficit irrigation strategies for sustaining vegetable production in water-limited regions: A Review Hort. Sci., 54:1872-1878.

Smith, C.J.; M.B. Peoples; G. Keerthisinghe and T.R. Jomes (1994). "Effect of surface applications of lime, gypsum and Phosphorgypsum on the alleviating of surface and sub-surface acidity in soil under pasture. Australian Journal of Soil Research. 32 this, (5): 995. ISSN 004- 9573.

Snedecor, G.W. and W.G. Cochran (1980). Statistical Methods. (7th ed.) Iowa State Univ. Iowa, U.S.A.

Stakman, W. P. and G. G. Vanderhast (1962). The use of the pressure membrane apparatus to determine soil moisture constants at P.F 3.0 to 4.2 inclusive. Inst. for Land and Water Manag Res., Note No. 139.

Taha, A. A. ; M. A. Ibrahim ; A. M. Mosa and M. N. EL-Komy (2017). Water productivity of wheat crop as affected by different sowing dates and deficit irrigation treatments. J.Soil Sci. and Agric. Eng., Mansoura Univ., 8: 521 - 529.

Tojo Soler, C.M. ; A. Suleiman ; J. Anothai ; I. Flitcroft and G. Hoogenboom (2013). Scheduling irrigation with a dynamic crop growth model and determining the relation between simulated drought stress and yield for peanut. Irrig. Sci., 31:889-901 
Usman, A.; Y. Kuzyakov and K. Stahr (2005). Effect of clay minerals on immobilization of heavy metals and microbial activity in a sewage sludge contaminated soil. Journal of Soils and Sediments, 5: 245-252.

Zaman, R. ; A.R. Akanda ; S.K. Biswas and M.R. Islam (2017). Effect of deficit irrigation on raised bed wheat cultivation. Cercetări Agronomice în Moldova,4 (172): 17-28.

Zayton, A.M. ; A.E. Guirguis and Kh. A. Allam (2014). Effect of sprinkler irrigation management and straw mulch on yield, water consumption and crop coefficient of peanut in sandy soil. Egypt. J. Agric. Res., 92:657-673.

$$
\begin{aligned}
& \text { تأثير معاملات الرى و بعض محسنات التربة على خواص الارض و انتاجية } \\
& \text { محصولى القمح و الفول السودانى فى الارض الرملية }
\end{aligned}
$$

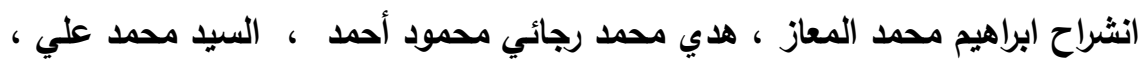

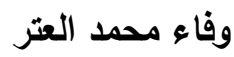

$$
\begin{aligned}
& \text { معهز بحوث الأراضى والمياة والبيئة - مركز البحوث الزراعية - الجيزة - مصر مدر }
\end{aligned}
$$

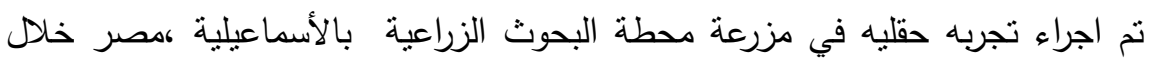

$$
\begin{aligned}
& \text { موسمى (2014 و 2015). نم زراعه القمح صنف (جيزة 168) في موسم الثتاء2014 تحت }
\end{aligned}
$$

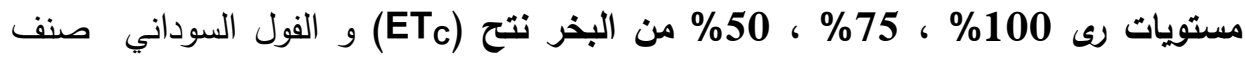

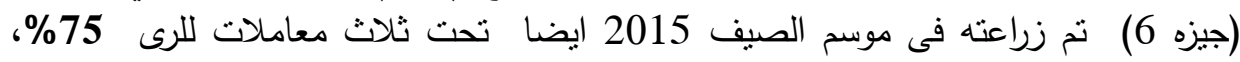

$$
\begin{aligned}
& \text { 50\%50 250 استنفاذ مستوى رطوية التربة (ASMD) من اجمالى الماء الميسر الكلى } \\
& \text { وللتربة. } \\
& \text { و كانت المعاملات كما يلي :- } \\
& \text { كمنترول } \\
& \text { - } \\
& \text { FYM }-3 \\
& \text { و } \\
& \text { وكانت النتائج كما يلى اكلى: }
\end{aligned}
$$

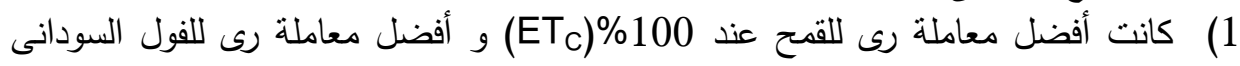

$$
\begin{aligned}
& \text { كانت عند مقارنة" بالمعاملات الاخرى و الكنترول. }
\end{aligned}
$$




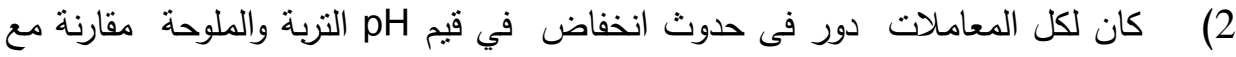

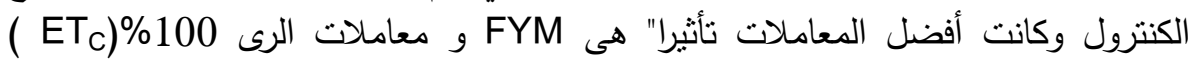

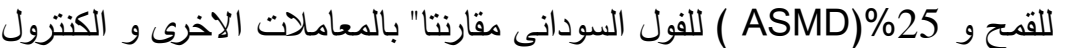

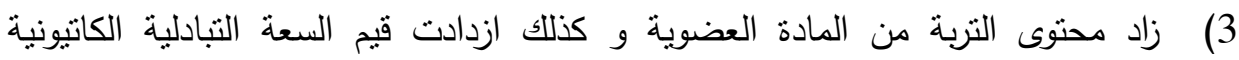
بأستخدام كل المعاملات بالمقارنة مع الكنادة الكنترول.

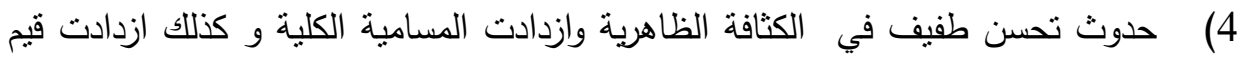

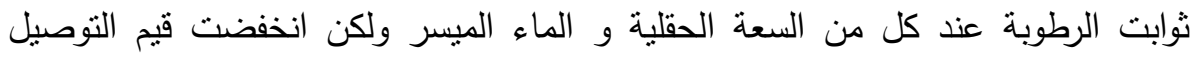

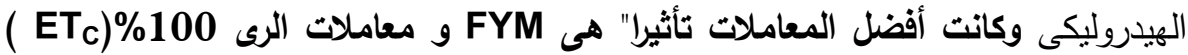
للقمح و ASMD) \%25 ) للفول السودانى مقارنة" بالمعاملات الاخرى و الكنترول.

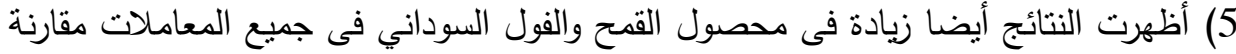

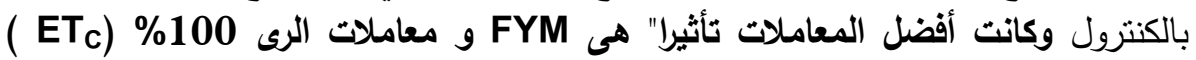

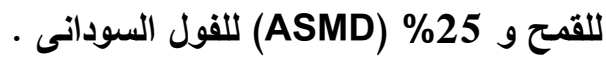

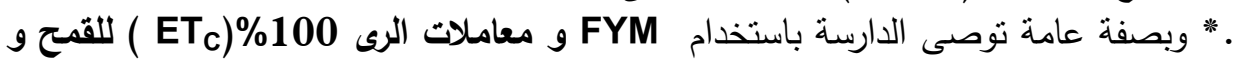
للفول السودانى لأن هذه المعاملات تعمل على تحسين خواص الاصل الارض (ASMD)\%25 الكيميائية و الطبيعية وبالتالي زيادة محصولى القمح و الفول السوداني في الأراضى الرملية. 